\title{
Propuesta de Ecuaciones Predictivas de Capacidad Inspiratoria y Flujo Espiratorio Máximo Considerando Mediciones Torácicas: Un Estudio Piloto
}

\author{
Proposal of Predictive Equations of Inspiratory Capacity and Maximum \\ Spiratory Flow Considering Thoracic Measurements: A Pilot Study
}

Rodrigo Muñoz Cofréé \& Mariano del Sol ${ }^{2}$

MUÑOZ, C. R. \& DEL SOL, M. Propuesta de ecuaciones predictivas de capacidad inspiratoria y flujo espiratorio máximo considerando mediciones torácicas: un estudio piloto. Int. J. Morphol., 36(1):333-337, 2018.

RESUMEN: El objetivo del siguiente estudio fue determinar si las mediciones de tórax provocan modificaciones en las ecuaciones predictivas de capacidad inspiratoria (CI) y flujo espiratorio máximo (FEM). Se evaluaron 24 sujetos de sexo masculino entre 18 y 26 años, todos estudiantes sedentarios de la Universidad Católica del Maule, Chile. Se les realizó antropometría corporal básica (peso y talla corporal) y específica de tórax, diámetro antero-posterior (DAT) y transverso (DTT) de tórax y perímetro mesoesternal (PME). Posterior a esto, se evaluó la función ventilatoria a través de pletismografía corporal. Para estimar las ecuaciones de regresión lineal se utilizó el método de mínimos cuadrados relacionando la CI y FEM. La comparación entre el valor predicho establecido y nuestra propuesta fue comparado utilizando la prueba t de student o U de Mann-Whitney según correspondiera, considerándose un nivel de significancia estadística de $\mathrm{p}<0,05$. Los resultados indican que para la propuesta de la ecuación de FEM se consideró el DTT y PME, logrando diferencias significativas con los valores de Knudson et al. Por tanto, se concluye que las mediciones de DTT a VR y PME a CPT influyeron en la ecuación predictiva de FEM en estudiantes sedentarios.

PALABRAS CLAVES: Antropometría de tórax, función pulmonar, ecuaciones predictivas.

\section{INTRODUCCIÓN}

En Chile, las enfermedades del sistema respiratorio constituyen el tercer grupo de causas de muerte, al mismo tiempo son la segunda causa de egreso hospitalario en sujetos mayores de 65 años (Olmos et al., 2015). Por tanto un diagnóstico oportuno, es vital para la disminución de estas cifras, así, una correcta valoración de la función pulmonar contribuiría a lograr este objetivo.

En un gran número de laboratorios de función pulmonar locales se utilizan los valores referenciales de Knudson et al. al no contar con parámetros nacionales (Alvarez et al., 2004). En este contexto, Gutiérrez et al. (2014) definieron ecuaciones predictivas de función pulmonar en población chilena sana, en donde los valores derivados de estas fueron significativamente superiores a los de Knudson et al. Por tanto, los investigadores sugieren la obtención de valores de referencia a partir de sujetos sanos, con características antropométricas similares a la población blanco.
Si bien existen numerosos factores que influyen en los valores de función pulmonar, se reconocen como los más importantes al sexo, edad, etnia y antropometría (Whittaker et al., 2005). Histórica y operacionalmente se ha utilizado la edad, altura y peso para determinar valores referenciales, sin embargo, en la actualidad se sabe que la longitud de los miembros inferiores en relación al tórax son diferentes en distintas poblaciones, por lo que la altura no representaría exactamente la participación del tórax en el funcionamiento del proceso de ventilación (Menezes et al., 2015).

Existe evidencia de que las diferencias en las dimensiones del tórax en adultos pueden generar una disparidad en la función pulmonar (Whittaker et al.). Para explicar estos contrastes, las características del tamaño del cuerpo han sido exploradas, no así factores específicos de la caja torácica como el tejido graso y la masa magra que la rodean o sus diámetros y longitudes, los cuales podrían influir en una co- 
rrecta función pulmonar, por lo que existen probabilidades de que estos factores contribuyan a explicar tales diferencias (Donnelly et al., 1991). Torres Santibáñez et al. (2017) estudiaron en jóvenes chilenos la relación entre diámetro y perímetro de tórax y su función pulmonar, observaron en el grupo sedentario, correlaciones de buenas a excelentes entre diámetro y perímetro de tórax con capacidad inspiratoria (CI) y presión inspiratoria máxima, respectivamente.

Específicamente, la CI ha demostrado tener una relación mayor que el volumen espiratorio forzado al primer segundo $\left(\mathrm{VEF}_{1}\right)$ con la capacidad de realizar ejercicio en paciente con EPOC. Por otra parte el flujo espiratorio máximo (FEM) puede determinar el estado de las vías aéreas de alto calibre, posibilitando el diagnóstico y monitorización de patologías respiratorias de manera rápida y confiable. De esta manera, el objetivo del presente trabajo fue determinar si las mediciones de tórax provocan modificaciones en las ecuaciones predictivas de CI y FEM.

\section{MATERIAL Y MÉTODO}

Sujetos: Correspondieron a 24 estudiantes entre 18 y 26 años todos de la Universidad Católica del Maule Talca, Chile, grupo control reclutado para la investigación de Torres Santibáñez et al. Los criterios de exclusión fueron; presentar enfermedad respiratoria crónica o aguda al momento de la evaluación, tener hábito tabáquico y evidenciar deformidades en la caja torácica. Este estudio fue aprobado por el Comité de Ética Científica de la Universidad Católica del Maule.

Diámetros torácicos: El evaluado debió estar de pie, tórax descubierto y miembros superiores en posición de descanso. Se utilizó para esta medición un antropómetro mayor (Campbell RC-20, Rosscraft ${ }^{\circledR}$ S.R.L.). Se realizó una marca en el cuarto espacio intercostal por ambos planos sagitales y a nivel del esternón. Para el diámetro transverso (DTT) las ramas del antropómetro se ubicaron en las marcas sagitales. En el diámetro antero-posterior (DAT), las ramas del antropómetro se posicionaron por anterior sobre un punto meso-esternal y por posterior en el proceso espinoso que se encuentra a nivel con el punto anterior. Ambas mediciones las realizamos a capacidad pulmonar total (CPT) y volumen residual (VR) (International Society for Advancement of Kinanthropometry).

Perímetro mesoesternal (PME): El evaluado debió estar de pie, tórax descubierto y miembros superiores en posición de descanso, se utilizó una cinta ergonómica $\left(\mathrm{SECA}^{\circledR}\right.$ 201) para las mediciones. Se determinó como punto de re- ferencia la marca meso-esternal, se posicionó la cinta ergonómica en contacto total con la piel. Se realizó la medición a CPT y VR (International Society for Advancement of Kinanthropometry).

Evaluación de la función pulmonar: En todas las pruebas se midió según la normativa de la American Thoracic Society (Miller et al., 2005). Una vez registrada la edad, talla $\left(\right.$ SECA $\left.^{\circledR} 220\right)$ y peso corporal (SECA $\left.{ }^{\circledR} 840\right)$, el sujeto debió permanecer sentado y relajado al menos unos 10 minutos previos a la evaluación. Las pruebas se realizaron en un pletismógrafo corporal (Mediagraphics Modelo PlatinumElite $\mathrm{DL}^{\circledR}$ ).

Capacidad vital forzada (CVF): De manera breve, el sujeto ventiló a volumen corriente por cinco ciclos respiratorios a través del neumotacógrafo y posterior a esto realizaró una maniobra inspiratoria máxima y después una espiración máxima forzada. Se seleccionó la mejor prueba de un mínimo de tres maniobras aceptables y reproducibles (ATS, 2005).

Volúmenes pulmonares: Se ajustó la boquilla a la altura del paciente, el evaluado debió asegurar que su boca estuviera adosada a esta pieza durante toda la prueba para evitar fugas de aire, paralelamente con sus manos bloqueó la musculatura facial durante la evaluación. Posteriormente, se cerró la cabina y se le indicó realizar cuatro ventilaciones a volumen corriente. Se instruyó al sujeto "jadear suavemente" intentando mover volúmenes de entre 50 a $60 \mathrm{~mL}$, a una frecuencia cercana a las 60 repeticiones por minuto $(1 \mathrm{~Hz})$. El profesional a cargo activó el shutter durante 2 a 3 segundos, después de esto se indicó una inspiración máxima hasta CPT y de manera seguida una espiraciónpara llegar a VR (Compte et al., 2002).

Estadística: En el análisis de los resultados, se utilizaron las aplicaciones informáticas Microsoft Office Excel 2010 para tabular datos, SPSS $20^{\circledR}$ para el análisis estadístico y GraphPad Prism $5^{\circledR}$ para dibujar gráficos, los datos fueron presentados en Media y Desviación Estándar. Para estimar las ecuaciones de regresión lineal se utilizó el método de mínimos cuadrados relacionando la CI y el FEM con la edad en años, la estatura en metros, el peso en kilogramos, DAT, DAP y PME en centímetros. Se calculó el coeficiente de determinación (R2) y el error estándar de la estimación (SEE) como medidas de la calidad del ajuste de las ecuaciones estimadas, además se midió la significancia estadística de cada uno de los coeficientes con el objetivo de remover aquellos términos del modelo que no fueran significativos $(p>0,05)$. Los valores obtenidos de esta propuesta fueron sometidos a pruebas estadísticas inferenciales; primero se determinó la normalidad de los datos a través de la prueba Shapiro-Wilks. Dependiendo de la distribución de los datos se utilizó t de Student o la U de MannWhitney, se consideró un nivel de significancia de $\mathrm{p}<0,05$. 


\section{RESULTADOS}

El grupo estuvo compuesto por 24 estudiantes con una edad promedio de 21,37 $\pm 1,27$ años, el IMC indica que la muestra se encontraba en sobre-peso (Tabla I). Las pruebas de función ventilatoria se encontraron dentro de rangos de normalidad al ser comparadas con sus valores de referencia (Tabla II). Los modelos complejos de ecuaciones de regresión no demostraron una mejor capacidad de predicción que la obtenida de las ecuaciones lineales simples, según lo evaluado por el ajuste coeficiente de correlación múltiple, además cada una de las ecuaciones lineales cumplieron con los supuestos del análisis estadístico (independencia y homoscedasticidad) para todos los intervalos de variables predictoras, estás se observan en la Tabla III. Al comparar los resultados derivados de las ecuaciones propuestas versus las recomendadas los resultados fueron; en

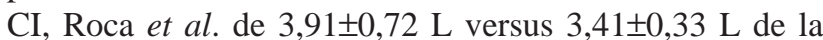
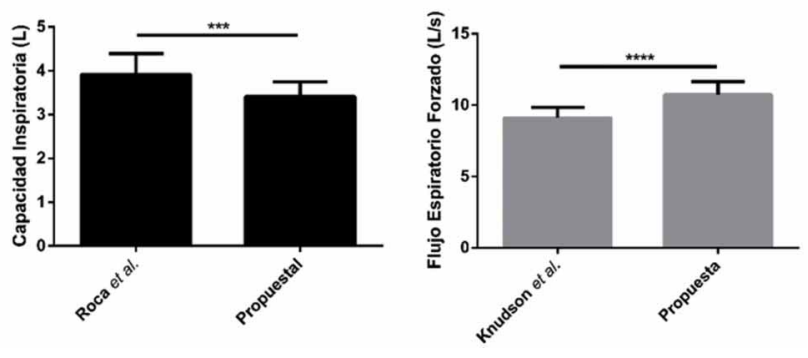

Fig. 1. Comparación de los valores de CI y FEM según recomendación y propuesta en estudiantes sedentarios de la Universidad Católica del Maule. $* * *$ : $p<0,001 ; * * * *$ : $<0,0001 ; \mathrm{L} / \mathrm{s}$ : litros por segundo.

Tabla I. Antropometría de estudiantes sedentarios.

\begin{tabular}{lc}
\hline Variable & Valor \\
\hline Edad (años) & $21,37 \pm 1,27$ \\
Peso $(\mathrm{kg})$ & $72,04 \pm 9,67$ \\
Talla $(\mathrm{m})$ & $1,75 \pm 0,08$ \\
IMC $\left(\mathrm{kg} / \mathrm{m}^{2}\right)$ & $23,5 \pm 1,52$ \\
Talla Sentado $(\mathrm{cm})$ & $91,94 \pm 3,65$ \\
\hline Diámetro Tórax Transverso CPT $(\mathrm{cm})$ & $32,23 \pm 1,90$ \\
Diámetro Tórax Transverso VR $(\mathrm{cm})$ & $29,28 \pm 1,45$ \\
Diámetro Antero-posterior CPT $(\mathrm{cm})$ & $21,35 \pm 1,66$ \\
Diámetro Antero-posterior VR $(\mathrm{cm})$ & $18,68 \pm 1,68$ \\
Perímetro Meso-esternal CPT $(\mathrm{cm})$ & $97,29 \pm 4,30$ \\
Perímetro Meso-esternal VR $(\mathrm{cm})$ & $92,36 \pm 5,93$ \\
\hline
\end{tabular}

CPT: capacidad pulmonar total; VR: volumen residual. ecuación propuesta, con un valor $\mathrm{p}<0,001$. Y en el FEM, Knudson et al. 9,10 $\pm 0,72 \mathrm{~L} / \mathrm{s}$ en relación a 10,73 \pm 0,91 L/ $\mathrm{s}$ de la ecuación propuesta, $\mathrm{p}<0,0001$ (Fig. 1).

Tabla II. Función ventilatoria de estudiantes sedentarios.

\begin{tabular}{lc}
\hline Variable & Valor \\
\hline CVF (L) & $5,43 \pm 0,7$ \\
Predicho (L) & $4,99 \pm 0,51$ \\
VEF1 (L) & $4,64 \pm 0,6$ \\
Predicho (L) & $4,30 \pm 0,45$ \\
VEF $_{1} / C V F(\%)$ & $85,75 \pm 5,35$ \\
Predicho & $86,20 \pm 0,72$ \\
FEF 25 (L/s) & $8,79 \pm 1,23$ \\
Predico & $8,34 \pm 0,44$ \\
FEF 75 (L/s) & $2,88 \pm 1,18$ \\
Predicho (L/s) & $2,44 \pm 0,24$ \\
FEF $25-75$ (L/s) & $4,93 \pm 1,12$ \\
Predicho (L/s) & $4,81 \pm 0,48$ \\
FEF máx (L/s) & $10,24 \pm 1,37$ \\
Predicho (L/s) & $9,10 \pm 0,72$ \\
CVIF (L) & $4,74 \pm 0,84$ \\
Predicho (L) & - \\
\hline CVL (L) & $5,17 \pm 0,9$ \\
Predicho (L) & $4,99 \pm 0,51$ \\
CI (L) & $3,44 \pm 0,69$ \\
Predicho (L) & $3,91 \pm 0,47$ \\
VRE (L) & $1,83 \pm 0,54$ \\
Predicho (L) & $1,08 \pm 0,15$ \\
VR (L) & $2,11 \pm 0,57$ \\
Predicho (L) & $1,60 \pm 0,10$ \\
CPT (L) & $7,17 \pm 0,99$ \\
Predicho (L) & \\
\hline & \\
\hline
\end{tabular}

CVF: capacidad vital forzada; VEF1: volumen espiratorio forzado en el primer segundo $\mathrm{VEF}_{1} / \mathrm{CVF}$ : relación entre el volumen espiratorio forzado en el primer segundo y capacidad vital forzada; \%: porcentaje; FEF ${ }_{25}$ : flujo espiratorio forzado al $25 \%$ de la capacidad vital forzada; FEF ${ }_{25-75}$ : flujo espiratorio forzado entre el 25 y $75 \%$ de la capacidad vital forzada; $\mathrm{FEF}_{75}$ : flujo espiratorio forzado al $75 \%$ de la capacidad vital forzada; FEF máx: flujo espiratorio forzado máximo; L/s: litros partidos por segundos; CVIF: capacidad vital inspiratoria forzada; L: litros; s: segundos; CVL: capacidad vital lenta; CI: capacidad inspiratoria, VRE: volumen de reserva espiratoria; VR: volumen residual; CPT: capacidad pulmonar total; L: litros. Valor $\mathrm{p}$ indica si existe o no diferencia significativa *: $\mathrm{p}<0,05, * * * \mathrm{p}<0,001$. 
MUÑOZ, C. R. \& DEL SOL, M. Propuesta de ecuaciones predictivas de capacidad inspiratoria y flujo espiratorio máximo considerando mediciones torácicas: un estudio piloto Int. J. Morphol., 36(1):333-337, 2018

Tabla III. Descripción de las pruebas de función ventilatoria de la muestra estudiada en nadadores y sedentarios.

\begin{tabular}{lllr}
\hline Variable & Autor & \multicolumn{1}{c}{ Ecuación } & $\mathrm{r}^{2}$ \\
\hline Capacidad & Roca et al. & $41-245($ talla)+65*0,005*IMC-5073 & 0,48 \\
Inspiratoria & Propuesta & $0,36+0.018[$ peso(kg) $]+1,020[$ talla corporal $(\mathrm{m})] \pm 0,34$ & 0,46 \\
Flujo Espiratorio & Knudson et al. & $0,094 *($ talla en $\mathrm{cm})-[0,035($ edad en años)] $-5,993$ & 0,64 \\
Máximo & Propuesta & $4,889-0,55[$ tórax transverso VR $(\mathrm{cm})]+0,231[($ Perímetro Mesoesternal CPT $(\mathrm{cm})] \pm 0,47$ & 0,62 \\
\hline
\end{tabular}

VR: volumen residual; CPT: capacidad pulmonar total.

\section{DISCUSIÓN}

El objetivo del presente trabajo fue determinar si las mediciones de tórax generan modificaciones en las ecuaciones predictivas de la CI y el FEM en estudiantes sedentarios de la Universidad Católica del Maule. Los resultados indican que la inclusión de las dimensiones torácicas generaron cambios significativos en la variable flujo (Fig. 1). Numerosos factores influyen en los valores de función pulmonar, históricamente los más estudiados han sido el sexo, la edad, la antropometría y la etnia (Gutiérrez et al.). Otros como la masa magra, diámetros y longitudes de tórax, se cree que también podrían predecir los valores de función pulmonar, sin embargo, debido a su baja factibilidad clínica han sido escasamente investigados (Menezes et al.; Donnelly et al.).

Tanto la altura como la forma del tórax pareciera ser un factor determinante en las mediciones de la CPT, aquí los caucásicos tienen tórax más anchos y altos, además de mayor altura corporal (Donnelly et al.). A mayor tamaño de la caja torácica mayor CPT, situación que incide sobre los volúmenes y flujos pulmonares, además de asociarse a la fuerza de los músculos inspiratorios, conformación de los pulmones y de la pared del tórax (Peters et al., 2017). Específicamente, las diferencias observadas en las ecuaciones obtenidas, se explicarían en su gran mayoría por la altura y por el diámetro del tórax, situación concordante a lo reportado por Donnelly et al.

La función pulmonar es proporcional al tamaño del cuerpo y tiene una relación directa con la altura en posición bípeda, así lo indican los resultados reportados por Donnelly et al. y Gutiérrez et al. Complementa-riamente, se ha observado una menor función pulmonar en estratos socioeconómico bajos, debido a una disminución del desarrollo corporal en los primeros años de vida, situación que se mantiene hasta la edad adulta (Menezes et al.). Esto concuerda parcialmente con los resultados encontrados, donde la altura solo fue significativa para la CI y no para el FEM.

Si bien la altura es un predictor importante de la función pulmonar (Gutiérrez et al.; Lisboa et al., 2007; Donnolly et al.), las dimensiones del tórax podrían ser mejores que la talla en bípedo (Donnolly et al.; Peters et al.). En el caso del FEM se utilizó, debido a su significancia estadística, el DTT a VR y el PME a CPT, esto tendría su explicación a través de la biomecánica de la prueba utilizada para medir esta variable; i) en una primera etapa se inspira hasta CPT; ii) posteriormente se produce un cierre glótico asociado a una contracción isométrica de los músculos espiratorios, aumentando las presiones intra-abdominal e intra-torácica; iii) consecuencia de esto, se produce un flujo espiratorio de alta velocidad, 11 a $12 \mathrm{~L} / \mathrm{s}$, hasta llegar a VR (McCool, 2006). Por tanto, queda claramente establecido que los volúmenes utilizados en el punto uno y tres concuerdan con las mediciones de PME y DTT, respectivamente. Es importante destacar que las ecuaciones predictivas obtenidas por Donnelly et al., en donde se incluyen diámetros torácicos, se realizaron sobre radiografías a diferencia de nuestras mediciones las cuales se realizaron directamente sobre el tórax. Hecho considerado como una debilidad, debido a que no se puede aislar la participación del tejido graso en la mecánica ventilatoria. Al respecto se sabe que la acumulación de grasa en el mediastino y cavidad torácica, provocan cambios en la fisiología ventilatoria, debido a los efectos mecánicos e inflamatorios de esta condición, elevando el diafragma, aumentando la presión pleural y disminuyen los volúmenes pulmonares (Peters et al.).

Los resultados reportados, desde las ecuaciones propuestas, demostraron que existió una disminución significativa de la CI y un aumento significativo del FEM con respecto a los valores predicho de Roca et al. y Knudson et al. respectivamente. Esto coincide con lo indicado por múltiples estudios locales (Gutiérrez et al.; Lisboa et al.), donde se recomiendan que cada país e incluso cada laboratorio, disponga de sus propias ecuaciones predictivas, y en ausencia de estas, la American Thoracic Society recomienda usar valores referenciales en donde exista algún tipo de proximidad con la población estudiada. Específicamente, Lisboa et al. establecieron valores de referencia de la CI en individuos sanos, de entre 50 y 87 años de edad y los compararon con los recomendados por la literatura. Encontraron una disminución significativa de esta variable con respecto a los valores de Roca et $a l$, si bien los resultados de este estudio piloto concuerdan con lo reportado por Lisboa et al., es necesario destacar que las ecuaciones propuestas por este grupo integran las varia- 
bles de edad, peso y talla a diferencia de la nuestra, que solo cuenta con peso y talla, esta diferencia se debería a la muestra medida, la cual es de un rango etario estrecho (Tabla I), lo cual va en sentido opuesto a la representatividad que busca una ecuación predictiva. Del mismo modo, Gutiérrez et al. diseñaron ecuaciones espirométricas para población chilena, sus resultados indicaron que los valores de Knudson et al., subestimaban hasta en $320 \mathrm{~mL}^{2} \mathrm{VEF}_{\text {, }}$, si bien la variable de estudio de esta investigación fue el FEM, este es parte del $\mathrm{VEF}_{1}$. A diferencia de lo ocurrido con la $\mathrm{CI}$ en nuestra propuesta para el FEM se incluyó el DTT a VR y el PME a CPT, situación destacable, debido a que existe mayor evidencia que respalda la relación existente entre dimensiones torácicas y volúmenes pulmonares en lugar de esta con sus flujos (Donnolly et al.; Whittaker et al.; Peters et al.).

Esta investigación cuenta con limitaciones propias de un estudio piloto, el bajo tamaño muestral tiene como consecuencia una alta exigencia para obtener significancia estadística. Al mismo tiempo, el número de sujetos utilizados no responde a un cálculo muestral realizado para determinar ecuaciones predictivas sino a observar comportamiento de variables. Solamente se consideró al sexo masculino por tanto se desconoce la conducta de estas variables en el sexo femenino. A partir de la evidencia encontrada, sería de gran interés contar con exámenes imagenológicos para descartar la influencia del tejido graso en los valores obtenidos. Por último concluimos que las mediciones de DTT a VR y PME a CPT influyeron en la ecuación predictiva de FEM en estudiantes sedentarios mostrando diferencias significativas con los valores de Knudson et al.

MUÑOZ, C. R. \& DEL SOL, M. Proposal of predictive equations of inspiratory capacity and maximum spiratory flow considering thoracic measurements: a pilot study. Int. J. Morphol., 36(1):333-337, 2018.

SUMMARY: The aim of the following study was to determine if chest measurements cause changes in the predictive equations of inspiratory capacity (IC) and peak expiratory flow (PEF). Twenty- four male subjects between 18 and 26 years old, all sedentary students of the Universidad Catolica del Maule, Chile, were evaluated. They were subjected to basic body anthropometry (weight and body size) and chest specificity, anteroposterior diameter (APD) and transverse (TTD) of the thorax and mesosternal perimeter (MSP). After this, the ventilatory function was evaluated through body plethysmography. To estimate the linear regression equations, the least squares method were used, relating IC and PEF. The comparison between the established predicted value and our proposal was compared using the student t-test or Mann-Whitney U test as appropriate, considering a level of statistical significance of $\mathrm{p}$ $<0.05$. The results indicate that the TTD and MSP were considered for the proposal of the PEF equation, achieving significant differences with the values of Knudson et al. Therefore, it is concluded that measurements of TTD to RV and MSP to TPC influenced the predictive equation of PEF in sedentary students.

KEY WORDS: Chest anthropometry; Lung function; Predictive equations.

\section{REFERENCIAS BIBLIOGRÁFICAS}

Alvarez, G. C.; Brockmann, V. P.; Bertrand, N. P.; Caussade, L. S.; Campos, M. E. \& Sánchez, D. I. Aplicación clínica de los valores de referencia de espirometría realizados en niños chilenos. Rev. Med. Chile, 132(10):120510,2004

Compte, L.; Macián, V.; Blanco, M. \& Rodríguez, M. Manual SEPAR de Procedimientos, Volúmenes pulmonares. Madrid, Novartis Farmacéutica, 2002.

Donnelly, P. M.; Yang, T. S.; Peat, J. K. \& Woolcock, A. J. What factors explain racial differences in lung volumes? Eur. Respir. J., 4(7):829-38, 1991.

Gutiérrez, C. M.; Valdivia, C. G.; Villarroel, D. L.; Contreras, T. G.; Cartagena, S. C. \& Lisboa, B. C. Proposición de nuevas ecuaciones para calcular valores espirométricos de referencia en población chilena adulta. Sociedad Chilena de Enfermedades Respiratorias (SER). Rev. Med. Chile, 142(2):143-52, 2014.

International Society for Advancement of Kinanthropometry. International standards for anthropometric assessment. Potchefstroom. The International Society for the Advancement of Kinanthropometry, 2001.

Lisboa, C.; Leiva, A.; Pinochet, R.; Repetto, P.; Borzone, G. \& Díaz, O. Valores de referencia de la capacidad inspiratoria en sujetos sanos no fumadores mayores de 50 años. Arch. Bronconeumol., 43(9):485-9, 2007.

McCool, F. D. Global physiology and pathophysiology of cough: ACCP evidence-based clinical practice guidelines. Chest, 129(1 Suppl.):48S53S, 2006.

Menezes, A. M.; Wehrmeister, F. C.; Hartwig, F. P.; Perez-Padilla, R.; Gigante, D. P.; Barros, F. C.; Oliveira, I. O.; Ferreira, G. D. \& Horta, B. L. African ancestry, lung function and the effect of genetics. Eur. Respir. J., 45(6):1582-9, 2015.

Miller, M. R.; Hankinson, J.; Brusasco, V.; Burgos, F.; Casaburi, R.; Coates, A.; Crapo, R.; Enright, P.; van der Grinten, C. P.; Gustafsson, P., Jensen, R.; Johnson, D. C.; MacIntyre, N.; McKay, R.; Navajas, D.; Pedersen, O. F.; Pellegrino, R.; Viegi, G.; Wanger, J. \& ATS/ERS Task Force. Standardization of spirometry. Eur. Respir. J., 26(2):319-38, 2005.

Olmos, C.; Mancilla, P.; Martínez, L. \& Astudillo, P. Epidemiología de las consultas respiratorias de adultos en Santiago de Chile desde 2003 a 2008. Rev. Med. Chile, 143(1):30-8, 2015.

Peters, U.; Suratt, B. T.; Bates, J. H. T. \& Dixon, A. E. Beyond BMI: Obesity and lung diseases. Chest, 2017. doi: 10.1016/j.chest.2017.07.010 [Epub ahead of print].

Torres Santibáñez, C.; Parada Alarcón, R.; Medina González, P.; Escobar Cabello, M.; Escobar Inostroza, J. \& Muñoz Cofré, R. Toracic morphometry in the swimmer and its relation to the pulmonary function. Int. J. Morphol., 35(3):845-51, 2017.

Whittaker, A. L.; Sutton, A. J. \& Beardsmore, C. S. Are ethnic differences in lung function explained by chest size? Arch. Dis. Child. Fetal Neonatal Ed., 90(5):F423-8, 2005.

Dirección para Correspondencia:

Rodrigo Muñoz Cofré

Programa de Doctorado en Ciencias Morfológicas

Universidad de la Frontera

Av. Francisco Salazar 01145

Temuco - CHILE

Email: b.munoz13@ufromail.cl 